EDITORIAL

https://doi.org/10.19053/22160159.v8.n18.2017.7252

\title{
DEL ENSEÑAR Y EL APRENDER
}

La educación contemporánea requiere para lograr sus objetivos encontrar no solo la posibilidad de adaptación a los tiempos y contextos en los cuales está inmersa, sino, a la vez, resignificar los conceptos que han estado ligados a sus formas de actuación y procedimiento a lo largo de la historia. Reflexionar sobre el enseñar y el aprender parece una actividad connatural a los procesos educativos, pues las diferentes teorizaciones pedagógicas conducen a concepciones y posicionamientos diversos frente a estas prácticas. En el último tiempo se ha venido constituyendo un desplazamiento de la enseñanza por el aprendizaje, que ha producido grandes transformaciones en la manera como los sujetos contemporáneos se forman, y también en la manera como profesores e instituciones asumen en las nuevas dinámicas del conocimiento este rol.

Enseñar estuvo relacionado con la transmisión de contenidos de orden formativo, con la razón y el conocimiento como eje de la actividad, y con un sujeto que al poseer el saber lo comunica y controla la apropiación por parte de sus aprendices. Este ejercicio privilegia no solo las formas de la transmisión, la memoria, el contenido y la evaluación heterónoma como axioma, sino que a su vez posiciona una manera determinada de hacerlo. De otra parte, el aprender ha sido considerado, a diferencia del enseñar, como el ejercicio propio de los sujetos sobre los cuales actúa el enseñar. En este sentido, el aprender está situado en las estructuras de pensamiento, el proceso, la actividad y la posibilidad de confrontación de los conocimientos cotidianos con los científicos.

Estas dos formas de relativización tanto del enseñar como del aprender vuelven al escenario privilegiado de la discusión académica y pedagógica a propósito de los nuevos focos y contextos que las apuestas contemporáneas obligan. Es decir, se reviven preguntas como: ¿cuál es el sitio del enseñar y del aprender en las nuevas realidades? ¿'se puede privilegiar el uno sobre el otro? ¿Se requieren pensar conjuntamente en una relación con sus 
posibilidades y potencialidades concomitantes? ¿es necesario superar el binomio enseñanza-aprendizaje, sus efectos y sus prácticas?

Aunque las diferentes posturas teóricas y metodológicas contemporáneas apuestan por resoluciones de orden disciplinar específico, consideramos que esta discusión debe ser abordada y reactualizada con los presupuestos históricos que han involucrado la postura pedagógica en el sentido más clásico de la palabra y es su cercanía con los postulados de la paideia y la filosofía en el mismo sitio de emergencia como saber sistemático. En este sentido, el aprender y el enseñar no están separados de sus objetos de actuación, ni de la práctica concreta de la educación. El aprender y el enseñar se encuentran de tal manera que no pueden deslindarse, separarse o anularse.

Heidegger (2010) propone, en una serie de conferencias dictadas en la Universidad de Friburgo de Brisgovia, bajo el título genérico de ¿Qué significa pensar?, algunas pistas que pueden allanar este camino de relaciones y de nuevos significados entre el enseñar y el aprender. El filósofo plantea que el aprender está en relación directa con el pensar, en el sentido que aprender significa "poner el hacer y omitir en correspondencia con lo que en cada caso se nos da en lo esencial. Según el tipo de esencial [...] es diferente la manera de correspondencia y con ellos la forma de aprender" (p. 77). De aquí que, si el aprender es diferenciado y cada caso lleva diversas formas de acercamiento al mismo, se requiere que exista alguien dotado y preparado para enseñarlo. Pero ¿qué es enseñar en esta lógica del aprender? Para Heidegger (2010), enseñar "implica un hacer aprender [...] el auténtico maestro lo único que enseña es el arte de aprender" (p. 79). ¿Qué significa dejar aprender hoy? ¿Qué implica, o cuáles son los dispositivos y andamiajes teóricos y conceptuales que permiten este ejercicio, si el enseñar implica dejar aprender? ¿Cómo diferenciar los roles y las acciones educativas? ¿Quién define o quién legitima lo aprendido y lo enseñado?

Siguiendo la huella de Heidegger, Bárcena (2012) plantea que enseñar y aprender se convierten en acontecimientos que surgen de la necesidad de los sujetos, aunque afirma que la educación no es un hecho connatural en sí mismo al ser humano, quien, debido a una especie de caos y desorientación, acude al pensamiento, requiere aprender a pensar, educarse en el pensamiento, prestar atención y cuidar-se. En esta perspectiva, es necesario que el aprender y el enseñar den qué pensar y no se conviertan en un instrumento de competencia y reproducción; que en vez de servir 
para acumular contenidos de conocimiento, se conviertan en verdaderos acontecimientos de saber, es decir, la enseñanza, en la medida que es sugerente y da qué pensar, debe producir en su ejercicio momentos de saber y posibilidades de construir sentidos, y el aprender, en el ejercicio propio desarrollado por los sujetos, debe incluir atención, interés y cuidado.

De otra parte, las relaciones enseñar y aprender encuentran una especial disposición en el acompañamiento, el encuentro y la problematización. Deleuze (2002), afirma que

no aprendemos nada con aquel que nos dice; "haz como yo". Nuestros únicos maestros son aquellos que nos dicen: "haz junto conmigo", y que, en lugar de proponernos gestos que debemos reproducir supieron emitir signos susceptibles de desarrollarse en lo heterogéneo. (p. 52)

Esta idea nos remite a ubicar la acción del enseñar no en la reproducción, sino en la producción de signos, de formas que actuar sobre la sensibilidad del aprendiz, de acompañamiento y de caminar juntos, por eso "nunca aprendemos actuando como alguien, sino actuando con alguien, que no tiene relación de semejanza con lo que se aprende" (Deleuze, 1995, p. 32). En este sentido, enseñar siempre será más complejo y difícil que aprender, pues al implicar hacer aprender, hace que el enseñar como arte de aprender sea mostrar y no explicar (Ranciere, 2007), propiciar y no conducir, generar condiciones de posibilidad, y construir conjuntamente pautas de ubicación. Se convierte en un ejercicio geográfico (Gómez \& Pulido, 2016).

Así pues, las relaciones enseñar y aprender deben ser comprendidas no como formas de eficacia e instrumentalización, sino como posibilidades reactualizadas del pensamiento y la practica educativa. La perspectiva contemporánea ha ubicado a la pedagogía y a la didáctica como sitios de rendimiento y productividad, sobrevalorando el énfasis en la gestión, los indicadores y los resultados. Por eso, el enseñar y el aprender se han visto reducidos a cumplir los propósitos productivistas del mercado y las posibilidades laborales (Pulido, 2009). De aquí que el profesor intente ubicarse en el sitio del experto que planifica, ejecuta y controla un sinfín de actividades para lograr los propósitos anteriormente referidos. Se convierte en una especie de experto que soluciona y completa los vacíos. En contraposición, hoy se quiere posicionar una perspectiva centrada en el aprendizaje y las competencias que intenta alejarse del enseñar, como si el sujeto por sí mismo pudiese aprender el arte de aprender. Estas reflexiones invitan a leer en clave de enseñar y aprender los artículos publicados en el 
fascículo 18 de la revista Praxis \& Saber, para continuar generando líneas de investigación y posibilidades de acciones críticas en el sentido teórico y práctico que la educación contemporánea requiere. Este fascículo de Praxis \& Saber está integrado por once artículos de variadas apuestas teóricas e investigativas sobre la educación y la pedagogía.

El fascículo comienza con un grupo de artículos que presenta diversas formas de uso de la historia de la educación como forma y campo de la pedagogía contemporánea. En el primero, las profesoras Solyane Silveira Lima y Maria Zélia Maia de Souza, de la Universidades Federal de Bahía y Estatal de Rio de Janeiro (UERJ), presentan a consideración de los lectores los resultados de un proyecto de investigación que se titula Estrategias para o governo de crianças e jovens desvalidos no Brasil (1865-1905), que vincula las prácticas de dos instituciones públicas de asistencia y formación para el trabajo para niños pobres en el Brasil de final del siglo XIX y las relacionan con los procesos de educación formal. El profesor Alexánder Aldana Bautista de la Universidad Pedagógica Nacional comparte un trabajo titulado Cuerpos vestidos, apariencias aseadas y lujo maldecido: hacia una estética corporal en la escuela colombiana que utiliza como perspectiva metodológica el enfoque arqueológico-genealógico heredado del pensamiento de Foucault, y recrea, a partir de los uniformes escolares y los códigos en el vestir en las escuelas colombianas de finales del siglo XIX, la constitución de una nueva tecnología de gobierno a través de los discursos estetizantes. Otro de los trabajos presentados en la perspectiva de historia de le educación es el titulado Galapa: tu historia, mi presente, nuestro futuro del profesor Johnny Meca Ospina de la Universidad del Atlántico, quien presenta los resultados de un proyecto de investigación realizado con la comunidad del municipio de Galapa para reconocer los saberes tradicionales y cotidianos inmersos en la comunidad y plantear a partir de ellos una posibilidad de recuperación y transmisión de los mismos, aprovechando lo que le autor denomina zonas de contacto.

Un segundo grupo de artículos publicados en el presente fascículo responde a resultados de investigación y reflexión sobre la universidad. La profesora Diana Milena Peñuela de la Universidad Pedagógica Nacional presenta una parte de los resultados de su trabajo de tesis doctoral en el artículo titulado Antropotécnicas y dimensión del homo ludens en la educación superior en Colombia, el cual, con soporte en la categoría metodológica antropotécnica del filósofo alemán Peter Sloterdijk, describe las prácticas universitarias de extensión y bienestar universitario, lo cual permite una novedosa lectura de 
la universidad colombiana a lo largo del siglo XX, en medio de ejercicios de obediencia, contención y moral católica. Universidad y globalización: cambios y desafíos es el título de trabajo presentado por el profesor José Carvajal Sánchez de la Fundación Universitaria Juan de Castellanos, que plantea, a manera de reflexión, los escenarios, las posibilidades y los desafíos de la universidad que piensa en el presente, se inserta en él y desde allí resignifica su función. El autor parte de una sugestiva teoría denominada el Cisne Negro para leer el mundo universitario contemporáneo. Siguiendo con los temas sobre universidad, el profesor Juan Pablo Hincapié de la Universidad del Valle presenta Aproximación a la didáctica de la lectoescritura universitaria; dos reseñas a Jonathan Potter, un artículo arriesgado en su concepción y formulación que presenta como modo de estrategia didáctica la puesta en escena del ejercicio modélico de dos reseñas, una descriptiva y otra crítica, sobre la obra de Potter.

El tercer grupo de artículos presenta apuestas de orden metodológico concentradas en la obra de Michel Foucault. Los profesores Élver Chaparro de la Fundación Universitaria Juan de Castellanos y Pedro Alexander Sosa de la Universidad Pedagógica y Tecnológica de Colombia presentan un texto titulado Arqueología y genealogía, operadores conceptuales para investigar en educación, que analiza la caja de herramientas arqueológico-genealógica de la obra de Foucault como posibilidad para la investigación en educación y pedagogía, haciendo énfasis en la potencia y las debilidades de las mismas al momento de su utilización en este campo teórico de trabajo. En otro sentido y a partir de los desarrollos teóricos y metodológicos que propone la obra de Foucault en el periodo ético de su producción, la profesora Lesli Alexa Sánchez de la Universidad Pedagógica Nacional presenta un artículo de revisión titulado Lo vivo y la vida como prácticas de gobierno en la escuela.

Cambiando de escenario teórico e investigativo, Praxis \& Saber en su fascículo 18 publica dos trabajos relacionados con el aprendizaje y la enseñanza de las matemáticas. Los profesores Lida Esperanza Riscanevo y Alfonso Jiménez Espinosa ofrecen a nuestros lectores el trabajo titulado El aprendizaje del profesor de matemáticas como campo investigativo, que hace parte de un trabajo de tesis y muestra la relaciones entre experiencia y aprendizaje en la formación de los profesores de matemáticas en ejercicio a través de la constitución de comunidades de aprendizaje investigativo. En esta perspectiva teórica, se publica el trabajo de las profesoras Omaida Sepúlveda Delgado y Nelcy Rocío González Gutiérrez de la Universidad 
Pedagógica y Tecnológica de Colombia y del profesor Eliécer Aldana Bermúdez de la Universidad del Quindío titulado Estudio epistemológico del objeto Grupo: una mirada piagetiana a la luz del EOS, donde se aborda una análisis evolutivo sobre el objeto Grupo, realizado desde una mirada ontosemiótica del conocimiento y la instrucción matemática.

Para culminar este fascículo, se presentan dos trabajos. El primero es de los profesores Ana María Valle y Marco Antonio Jiménez de la Universidad Autónoma Nacional de México, titulado Lo educativo como experiencia fenomenológica, el cual intenta leer en clave filosófica la educación y lo que los autores conceptualizan como experiencia educativa, es decir, poder pensar su otredad. Por otro lado, está la entrevista realizada por la profesora Alba Nidia Triana al Dr. Eduardo Luis Menéndez bajo el título Educación médica, modelo hegemónico y atención primaria en salud.

Óscar Pulido Cortés

Editor

Leonor Gómez Gómer

Directora

\section{Referencias}

Bárcena, F. (2012). El aprendiz eterno. Filosofía, educación y el arte de vivir. Buenos Aires: Miño y Dávila.

Deleuze, G. (1995). Proust y los signos. Barcelona: Anagrama.

Deleuze, G. (2004). Diálogos. Barcelona: Pretextos.

Gómez, L, \& Pulido, O. (enero-mayo de 2016). La pedagogía su presente y su umbrales [Editorial]. Praxis \& Saber, 7(13), 9-14.

https://doi.org/10.19053/22160159.4157

Heidegger, M. (2010). ¿Qué significa pensar?. Editorial Trotta.

Pulido, Ó. (noviembre de 2009). Aprender y enseñar filosofía en el mundo contemporaneo. De la mercantilizacion del pensamiento al despliegue de su ejerecicio. Cuestiones de Filosofía, (11), 1-12

Ranciere, J. (2007). El maestro ignorante. Laertes. 